Check for updates

Cite this: RSC Adv., 2018, 8, 39849

Received 28th September 2018 Accepted 14th November 2018

DOI: $10.1039 / c 8 \mathrm{ra0} 0069 \mathrm{k}$

rsc.li/rsc-advances

\title{
Interactions of a paracyclophane-based conjugated oligoelectrolyte with biological membranes $\uparrow$
}

\author{
Jakkarin Limwongyut, (DD ${ }^{a}$ Yang Liu, ${ }^{c}$ Gayatri Shankar Chilambi, ${ }^{d}$ Thomas Seviour, ${ }^{d}$ \\ Jamie Hinks, ${ }^{d}$ Yuguang $\mathrm{Mu}^{\mathrm{c}}$ and Guillermo C. Bazan (D) *abe
}

\begin{abstract}
A three-dimensional conjugated oligoelectrolyte (COE) bearing a [2.2] paracyclophane unit (COE2-3-pCp) was synthesized. Its biological activity was determined both in vivo and in silico within the context of membrane perturbation and biocompatibility. Molecular dynamics simulations indicate that, compared to its linear analog (COE2-3C), COE2-3-pCp introduces more lipid disorder with higher extent of membrane thinning. COE2-3-pCp also exhibits a higher MIC towards E. coli K12 and yeast, while maintaining similar levels of membrane permeabilization. These findings suggest a new design of COEs as biocompatible cell permeabilizers.
\end{abstract}

\section{Introduction}

Conjugated oligoelectrolytes (COEs) are defined by a conjugated backbone with ionic pendant groups tethered by hydrocarbon

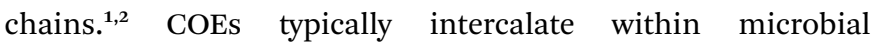
membranes in a well-organized fashion. ${ }^{3}$ Several molecular features are known to impact the extent of COE accumulation within cells and their effects on the membrane. For example, the degree to which the COEs intercalate within membranes depends on whether the charged groups are anionic or cationic. ${ }^{4}$ Additionally, the number of repeat units affects the distortion of the lipid bilayer and is a strong modulator of antimicrobial efficacy. ${ }^{5,6}$ Microbes with COEs have their properties and behaviour modified in ways that are desirable for specific applications. Consider, the homologous series of oligophenylenevinylene COEs illustrated by DSBN+, DSSN+ and COE1-5C (Scheme 1). While DSBN+ has a higher antimicrobial efficacy than DSSN+, both increase membrane permeability in E. coli $\mathrm{K} 12$. COE1-5C, in contrast, has been shown to rigidify membranes. ${ }^{5,7,8}$

Oligophenylenevinylene COEs studied thus far largely contain a planar, two-dimensional conjugated fragment. A

${ }^{a}$ Center for Polymers and Organic Solids, Department of Chemistry and Biochemistry, University of California, Santa Barbara, CA, 93106, USA. E-mail: bazan@chem.ucsb. edu

${ }^{b}$ Materials Department, University of California, Santa Barbara, CA, 93106, USA

${ }^{c}$ School of Biological Sciences, Nanyang Technological University, 637551, Singapore

${ }^{a}$ Singapore Centre on Environmental Life Sciences Engineering (SCELSE), Nanyang Technological University, 637551, Singapore

${ }^{e}$ School of Chemical and Biomedical Engineering, Nanyang Technological University, 637551, Singapore

$\dagger$ Electronic supplementary information (ESI) available: Experimental procedures, absorption and emission spectra, and NMR spectra. See DOI: 10.1039/c8ra08069k molecular structural variation that has yet to be considered concerns introducing dimensionality to the conjugated segment in order to understand possible repercussion of this structural modification on the reorganization of the membrane lipids and the overall perturbation of the bilayer. To accomplish this structural variation, we sought to include a rigid framework within the interior of the conjugated segment with an otherwise similar aspect ratio to phenylene unit. For this purpose, we chose the [2.2]paracyclophane (pCp) moiety. We hypothesized that three-dimensionality from the pCp adduct would increase

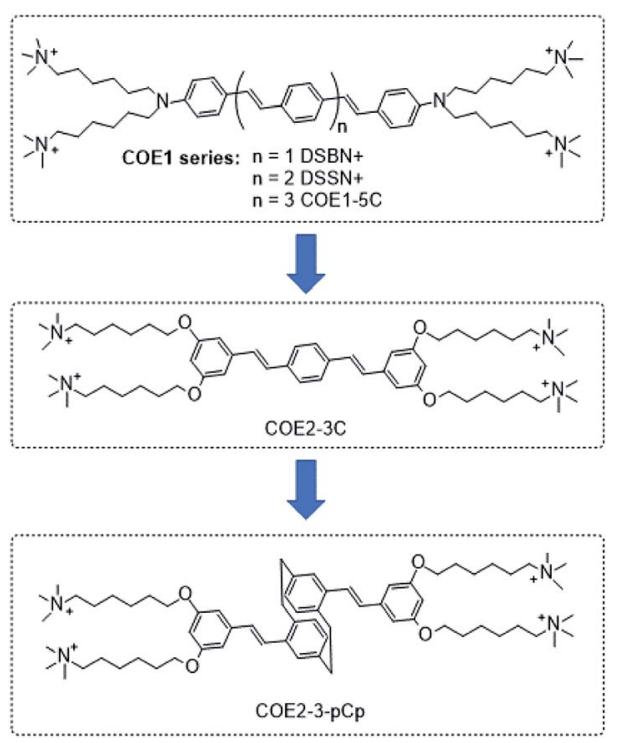

Scheme 1 Structural evolution from the COE1 series to the paracyclophane-based COE (COE2-3-pCp). lodide counterions are omitted from the structures. 
lipid disorder in the membrane. The structural evolution is illustrated in Scheme 1.

We compared the properties of COE2-3-pCp to those of its linear counterpart COE2-3C by first carrying out molecular dynamics simulations to elucidate deformations and disorder within a model lipid bilayer structure. The results of the simulations provide a framework for understanding observed differences in the modification of $E$. coli $\mathrm{K} 12$ brought about by membrane modification with additional context provided by biophysical experiments with vesicles and cells. Of interest is the finding that while COE2-3-pCp has a lower minimum inhibitory concentration (MIC) relative to COE2-3C, it can induce similar levels of membrane permeabilization. Implications of increased permeabilization for whole-cell biocatalysis have been previously investigated using $E$. coli $\mathrm{K} 12$ and yeast. ${ }^{7,9,10}$

\section{Results and discussion}

The synthesis of COE2-3-pCp is shown in Scheme 2. 4,16Dibromo[2.2]paracyclophane (1), a pseudo-para isomer of dibromo[2.2]paracyclophane, was used as the key starting material to maintain the overall linearity of the backbone. Compound 1 was converted into 2 by using $s$-BuLi, followed by addition of $\mathrm{N}, \mathrm{N}$-dimethylformamide (DMF). Formyl groups were then subjected to reduction and chlorination with sodium borohydride and thionyl chloride, respectively, to yield 4 . The intermediate $\mathbf{5}$ was synthesized using triethyl phosphite. Precursor 7 was prepared under Horner-Wadsworth-Emmons (HWE) reaction conditions from 5 and 6. Finally, treatment of 7 with trimethylamine provides COE2-3-pCp. COE2-3C, which is the planar analogue of COE2-3-pCp, was synthesized according to reported protocols. ${ }^{2,6}$

Absorption and fluorescence spectra of COE2-3-pCp are summarized in ESI (Fig. S1 $\dagger$ ). The UV-visible absorption spectrum of COE2-3-pCp in water shows three absorption peaks in the ultraviolet region, namely, $225 \mathrm{~nm}, 311 \mathrm{~nm}$ and a shoulder peak at $328 \mathrm{~nm}$. The COE2-3-pCp fluorescence exhibits an emission maximum at $436 \mathrm{~nm}$. The emission maximum exhibits a hypsochromic shift of $15 \mathrm{~nm}$ when treated with a solution of 1,2-dimyristoyl-sn-glycero-3-phosphocholine (DMPC) vesicles. In previous studies, it was shown that when COEs intercalate into lipid bilayers, fluorescence emission will shift to shorter wavelength due to the change of environment from a polar (water) to a non-polar (lipid tails) environment. Hence, the observed shift in the emission of COE2-3-pCp in the vesicles suggests that COE2-3-pCp similarly intercalates into lipid membranes. ${ }^{\mathbf{1 1}}$

To validate intercalation into lipid bilayers in microbes, $E$. coli $\mathrm{K} 12$ was treated with COE2-3-pCp in $50 \mathrm{mM}$ phosphate buffer saline (PBS) at the turbidity of $\mathrm{OD}_{600}=1$. After staining, cells were centrifuged at $7000 \mathrm{rpm}$ for 7 minutes and the supernatant was collected for UV-visible absorption measurements revealing more than $90 \%$ association of COE2-3-pCp to $E$. coli $\mathrm{K} 12$ cells, even when [COE2-3-pCp] $=40 \mu \mathrm{M}$ (Fig. 1a). Zeta potentials of cells and liposomes treated with COE2-3-pCp in $50 \mathrm{mM}$ PBS were also measured (Fig. 1b and c). An increase in zeta potential should be observed when COEs intercalate into the bilayers. However, while an increase was observed for liposomes, little change occurred with the cells (Fig. 1b). The same observations were observed for COE2-3C with E. coli K12. ${ }^{12} \mathrm{~A}$ plausible reason underlying the relatively constant zeta potential with $E$. coli may be the presence of the lipopolysaccharide (LPS) layer. With the thickness of the LPS being around 2-3 nm in $E$. coli $\mathrm{K} 12$, the COE cationic groups may not be able to reach the surface of the cells after their intercalation into the lipid bilayer due to the molecular length of the COEs. ${ }^{\mathbf{1 2 - 1 4}}$

To verify COE2-3-pCp intercalation, we examined liposomes made of $E$. coli total lipid extract, which lacks the LPS layer. As shown in Fig. 1c, the zeta potential increased with COE2-3-pCp concentration, indicating intercalation into the bilayer. Finally, to visually illustrate COE uptake, fluorescence signals in cell suspensions were imaged using two-photon microscopy. The images clearly show the accumulation of COE on the cells (Fig. 2).

Molecular dynamics (MD) simulations were undertaken on Gram-negative model membrane bilayer systems $(3: 1$ palmitoyloleoylphosphatidylethanolamine/
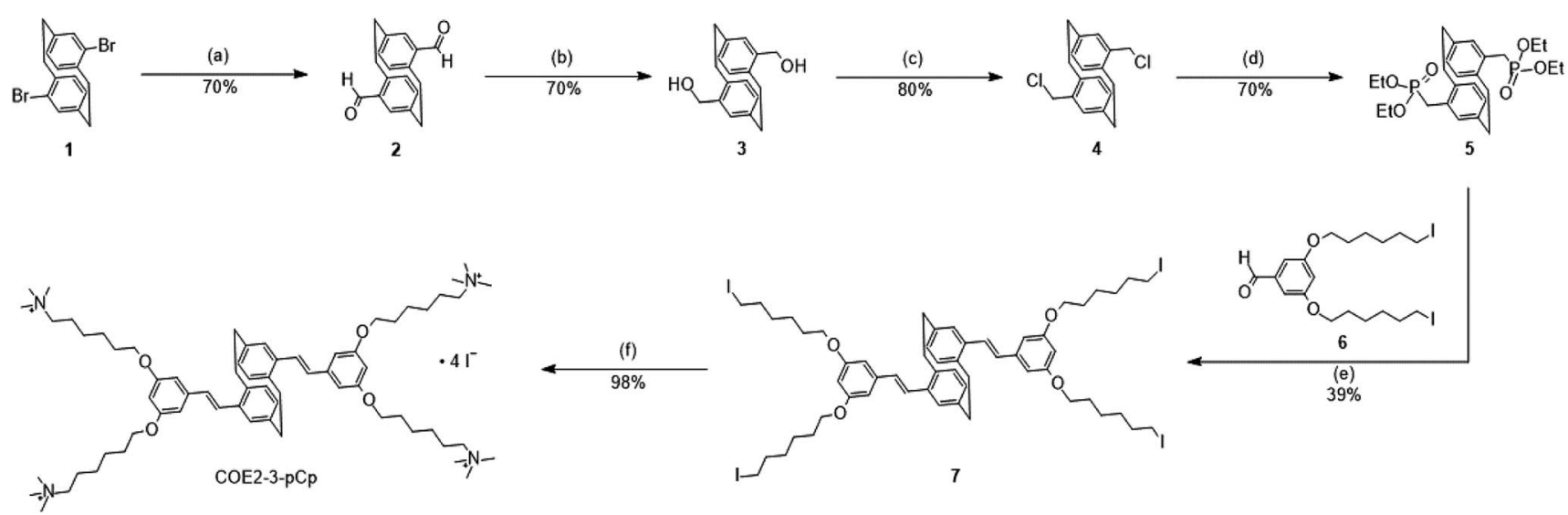

Scheme 2 Synthesis of paracyclophane-based COE (COE2-3-pCp). Reaction conditions: (a) s-BuLi (2.3 equiv.), DMF (10 equiv.), THF, $-78^{\circ} \mathrm{C}$ to rt, 3 h; (b) $\mathrm{NaBH}_{4}$ (3 equiv.), EtOH/THF (1 : 5), rt, 3 h; (c) $\mathrm{SOCl}_{2}$ (2.4 equiv.), MeCN, $50{ }^{\circ} \mathrm{C}, 5$ h; (d) P(OEt) ${ }_{3}, 135^{\circ} \mathrm{C}, 48$ h; (e) 6 (1.95 equiv.), NaO ${ }^{\mathrm{B} u}$ (2.1 equiv.), THF, rt, 6 h; (f) excess $\mathrm{NMe}_{3}, \mathrm{THF}, \mathrm{MeOH}, \mathrm{CHCl}_{3}, \mathrm{rt}, 48 \mathrm{~h}$. 


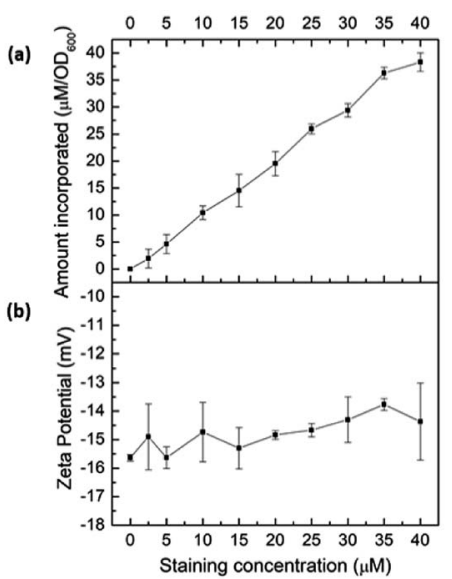

(c)

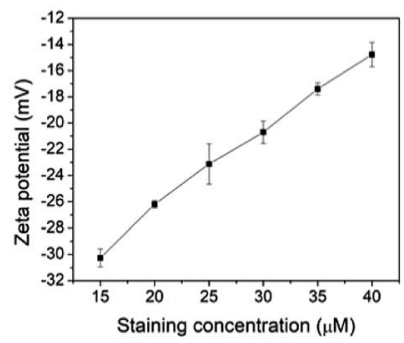

Fig. 1 (a) COE2-3-pCp association to E. coli K12 cells; zeta potential of (b) E. coli K12, and (c) E. coli total lipid extract vesicles at different COE2-3-pCp staining concentrations

palmitoyloleoylphospatidylglycerol, or POPE/POPG) doped with either COE2-3-pCp or COE2-3C (COE : POPE : POPG = $4: 110: 36)$. Relative to $\mathrm{COE} 2-3 \mathrm{C}, \mathrm{COE} 2-3-\mathrm{pCp}$ induced a greater degree of disorder, as illustrated by the more pronounced reduction in deuterium order parameter along the first fatty acid chain (sn1) of POPE predicted for COE2-3-pCp (Fig. 3a). The same effect was observed on POPG for both the sn1 and sn2 (i.e. second fatty acid) chain (Fig. S3†).

This increased disorder observed for COE2-3-pCp relative to COE2-3C resulted in a greater reduction of the average bilayer
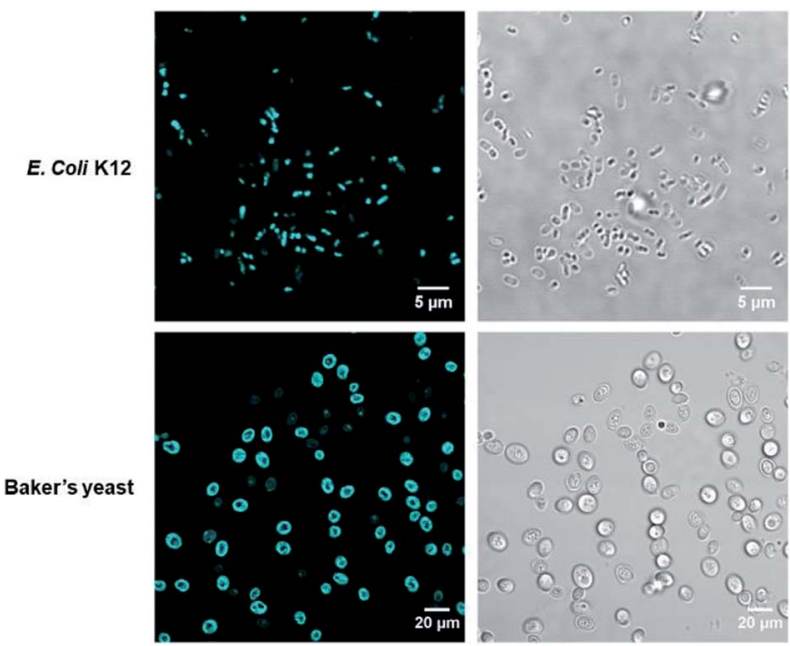

Fig. 2 Two-photon micrographs of $E$. coli $\mathrm{K} 12$ and yeast treated with COE2-3-pCp from fluorescence channel (left) and brightfield channel (right). The excitation wavelength was $725 \mathrm{~nm}$. thickness of the COE2-3-pCp-doped lipid bilayer (Fig. 3b). Earlier studies proposed that the COE length was a key parameter with regards to their antibiotic characteristics. Thus, antimicrobial activity was determined against E. coli $\mathrm{K} 12$ and yeast using broth microdilution method. ${ }^{15}$ All experiments were performed in triplicate (see ESI $\dagger$ ). Specifically, the minimum inhibitory concentrations (MICs) of COE2-3-pCp on E. coli K12 and yeast are $64 \mu \mathrm{M}$ and $128 \mu \mathrm{M}$, respectively, whereas those of COE2-3C are $16 \mu \mathrm{M}$ and $64 \mu \mathrm{M}$, respectively. Modification of the COE by introducing a three-dimensional moiety decreased inhibitory action despite reduction of bilayer thickness. Hinks et al. attributed a reduction in antimicrobial activity of a fluorinated COE to increased range of movement of the COE pendant arm, which reduced the mechanical shock to the lipid bilayer. Another effect of this increased lability was reduced molecular aggregation within the bilayer and it is possible that this also contributed to attenuating COE2-3-pCp driven membrane disruption. ${ }^{5}$

According to the probability distribution for intermolecular distance between each COE pair in a four COE simulation (see ESI $\dagger$ for definition) (Fig. 3c), there was a $36 \%$ reduction in the
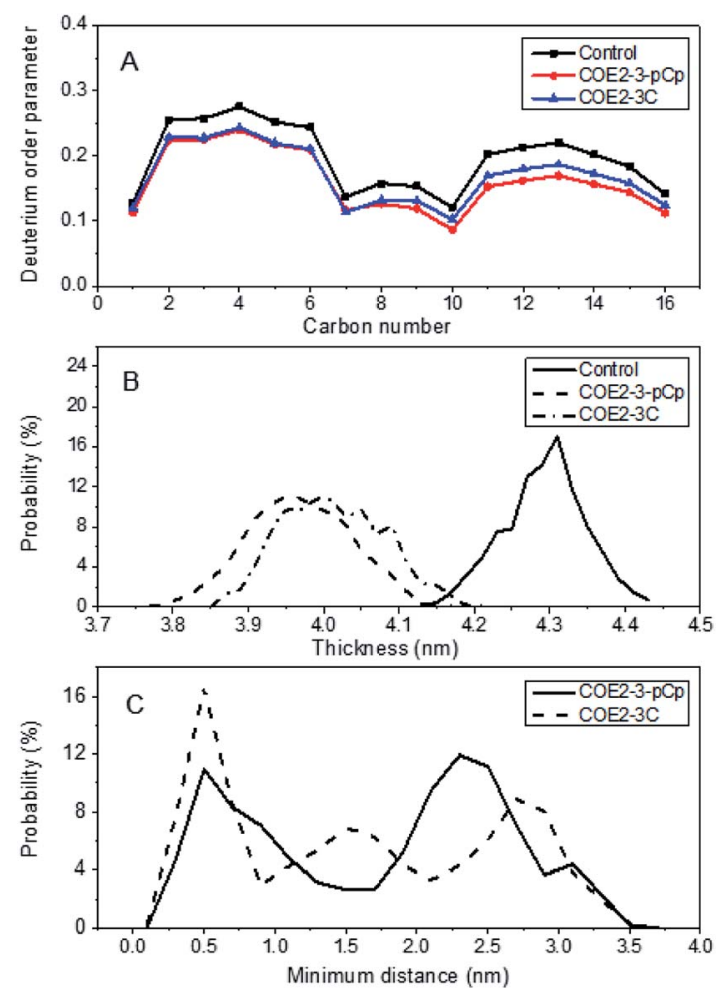

Fig. 3 (a) Local deuterium order parameter along the sn1 aliphatic chain of POPE for model bilayer system (122 : 40, POPE : POPG) only (i.e. control), and with either COE2-3-pCp or COE2-3C (4 : $110: 36$, COE : POPE : POPG). (b) Probability distribution function for average bilayer thickness for the model bilayer system only (122:40, POPE : POPG), and with either COE2-3-pCp or COE2-3C (4 : 110 : 36, COE : POPE : POPG) based on the center of mass distance between phosphorous atoms in the two leaflets. (c) Probability distribution function of minimum bridging distance between each pair of dopant molecules in model bilayer system simulations with either COE2-3pCp or COE2-3C (4:110:36, COE : POPE : POPG). The data were averaged across the last 50 ns of $3 \times 200$ ns simulations. 

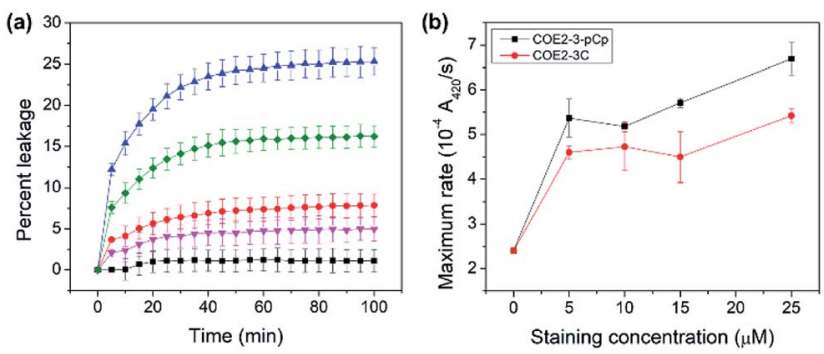

Fig. 4 (a) Leakage of calcein from E. coli total lipid extract vesicles over time (legend: $\mathbf{\Delta}=2.5 \mathrm{~mol} \%$ COE2-3-pCp, $-1 \mathrm{~mol} \%$ COE2-3$\mathrm{pCp}, \boldsymbol{\nabla}=2.5 \mathrm{~mol} \% \mathrm{COE} 2-3 \mathrm{C}, \boldsymbol{\nabla}=1 \mathrm{~mol} \% \mathrm{COE} 2-3 \mathrm{C}, \mathbf{\square}=$ control), (b) rate of ONPG hydrolysis at different staining concentrations in $E$. coli K12.

duration that COE2-3-pCp molecules exist as an aggregate, relative to $\mathrm{COE} 2-3 \mathrm{C}$, as determined from an intermolecular distance of $<0.5 \mathrm{~nm}$. While introducing a non-planar aspect to the design of COEs may increase disorder, MD simulations indicate that the effect of this with regards to antimicrobial activity is most reasonably offset by reduced aggregation.

COE permeabilization ability was determined by calcein released from $E$. coli total lipid extract vesicles treated with the two COEs. At the COE amount of $2.5 \mathrm{~mol} \%$ relative to lipids, COE2-3-pCp induced leakage up to $25 \%$ in 100 minutes, while COE2-3C induced $16 \%$ within the same timeframe (Fig. 4a). This supports the hypothesis that COE2-3-pCp has a potential to be a more effective membrane permeabilizer.

The impact of COEs on whole-cell biocatalysis was investigated using $E$. coli K12 hydrolysis of $o$-nitrophenyl $\beta$-D-galactopyranoside (ONPG) by $\beta$-galactosidase. ${ }^{16,17}$ Cells treated with COEs show increased catalytic rates. Turnover rates are 2.8 and 2.3-fold faster than untreated cells upon treatment with 25 $\mu \mathrm{M}$ COE2-3-pCp and COE2-3C, respectively (Fig. 4b). With the higher MIC of COE2-3-pCp compared to COE2-3C toward E. coli $\mathrm{K} 12$, the degree to which association with COEs impacts cell viability can thus be decoupled from the ability to permeabilize the membrane. Such an observation is surprising since these two properties usually follow the same trend. ${ }^{18}$

The membrane permeabilization effect was also tested with yeast. Relative extracellular ATP levels were used as an indicator of permeabilization as previously described. ${ }^{19}$ The amount of ATP in the supernatant is directly proportional to the observed luminescence signal from the luciferase-luciferin system. Yeast treated with $100 \mu \mathrm{M}$ of COEs show higher extracellular ATP than control, indicating permeabilization (Fig. 5a).

The enantioselective hydration of fumarate by fumarase was then chosen for a model system to monitor biocatalysis, as the turnover rate can be accelerated by increasing permeability. ${ }^{20}$ Yeast cells were treated with $25 \mu \mathrm{M}$ COEs per $0.1 \mathrm{~g}$ wet cells. Cells with COE2-3-pCp show significant catalytic acceleration from untreated cells at comparable degree to those treated with COE2-3C (Fig. 5b). Despite the slightly higher permeabilizing efficacy of COE2-3-pCp on E. coli, COE2-3-pCp is slightly less effective with yeast. Such differences may be related to the differences in membrane composition, but a more precise mechanistic rationale requires further work.
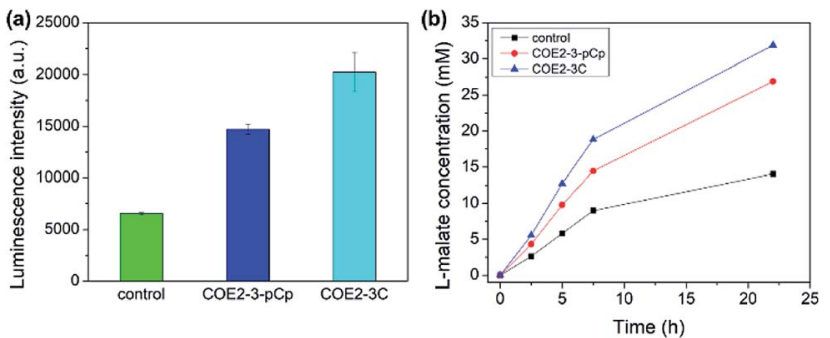

Fig. 5 (a) Luminescence intensity obtained from extracellular ATP assay with supernatants from yeast cells treated with the COEs, (b) concentration of L-malate produced by yeast over time. Yeast cells were stained with $25 \mu \mathrm{M}$ of the indicated COEs.

\section{Conclusions}

In summary, the paracyclophane-based COE2-3-pCp was designed and synthesized. Despite its similar length and cell association profile to its linear counterpart COE2-3C, COE2-3pCp exhibits a higher MIC towards E. coli $\mathrm{K} 12$ and yeast. Moreover, COE2-3-pCp permeabilizes lipid bilayers to a similar extent as COE2-3C and thus improves biocatalysis processes in both microorganisms relative to untreated cells. In contrast to the higher MIC of COE2-3-pCp compared to COE2-3C, MD simulation suggests the $\mathrm{pCp}$ unit introduces more lipid disorder with higher extent of membrane thinning. Perhaps more surprisingly, the general molecular topology of COE2-3$\mathrm{pCp}$ reduces the tendency to form aggregates in the membrane. To what degree self-association of COEs within the membrane impacts their ability to reduce cell viability remains an open question right now due to the absence of relevant experimental techniques. These insights hint to the strategic design of new COEs to manipulate intermolecular interactions that attenuate growth inhibition, while inducing permeabilization-findings that are relevant within the context of whole-cell biocatalysis.

\section{Conflicts of interest}

There are no conflicts to declare.

\section{Acknowledgements}

This work was supported by the Institute of Collaborative Biotechnologies through grant W911NF-09-0001 from the U.S. Army Research Office. We acknowledged the use of NRI-MCDB Microscopy Facility and the Multiphoton/Laser Scanning Confocal supported by the NCRR under Award \# 1S10RR02258501A1. SCELSE is funded by Singapore's National Research Federation, Ministry of Education, Nanyang Technological University (NTU), and National University of Singapore (NUS) and hosted by NTU in partnership with NUS. The work at SCELSE was supported by Singapore Ministry of Education through grant M4360005. J. L. is grateful for DPST fellowship from the Institute for the Promotion of Teaching Science and Technology, Thailand. 


\section{Notes and references}

1 Y. Wang, Y. Tang, Z. Zhou, E. Ji, G. P. Lopez, E. Y. Chi, K. S. Schanze and D. G. Whitten, Langmuir, 2010, 26, 12509-12514.

2 H. Hou, X. Chen, A. W. Thomas, C. Catania, N. D. Kirchhofer, L. E. Garner, A. Han and G. C. Bazan, Adv. Mater., 2013, 25, 1593-1597.

3 H. Yan, C. Catania and G. C. Bazan, Adv. Mater., 2015, 27, 2958-2973.

4 A. W. Thomas, C. Catania, L. E. Garner and G. C. Bazan, Chem. Commun., 2015, 51, 9294-9297.

5 J. Hinks, Y. Wang, W. H. Poh, B. C. Donose, A. W. Thomas, S. Wuertz, S. C. J. Loo, G. C. Bazan, S. Kjelleberg, Y. Mu and T. Seviour, Langmuir, 2014, 30, 2429-2440.

6 H. Yan, Z. D. Rengert, A. W. Thomas, C. Rehermann, J. Hinks and G. C. Bazan, Chem. Sci., 2016, 7, 5714-5722.

7 C. Catania, C. M. Ajo-Franklin and G. C. Bazan, RSC Adv., 2016, 6, 100300-100306.

8 J. Hinks, Y. Wang, A. Matysik, R. Kraut, S. Kjelleberg, Y. Mu, G. C. Bazan, S. Wuertz and T. Seviour, ChemSusChem, 2015, 8, 3718-3726.

9 R. F. Epand, J. E. Pollard, J. O. Wright, P. B. Savage and R. M. Epand, Antimicrob. Agents Chemother., 2010, 54, 3708-3713.
10 A. V. Presečki, B. Zelić and D. Vasić-Rački, Enzyme Microb. Technol., 2007, 41, 605-612.

11 L. E. Garner, J. Park, S. M. Dyar, A. Chworos, J. J. Sumner and G. C. Bazan, J. Am. Chem. Soc., 2010, 132, 10042-10052.

12 C. Catania, A. W. Thomas and G. C. Bazan, Chem. Sci., 2016, 7, 2023-2029.

13 M. Kastowsky, T. Gutberlet and H. Bradaczek, J. Bacteriol., 1992, 174, 4798-4806.

14 Q. Lu, J. Wang, A. Faghihnejad, H. Zeng and Y. Liu, Soft Matter, 2011, 7, 9366.

15 I. Wiegand, K. Hilpert and R. E. W. Hancock, Nat. Protoc., 2008, 3, 163-175.

16 R. E. Huber, G. Kurz and K. Wallenfels, Biochemistry, 1976, 15, 1994-2001.

17 H. E. Mostafa, K. J. Heller and A. Geis, Appl. Environ. Microbiol., 2002, 68, 2619-2623.

18 C. C. C. R. de Carvalho, Microb. Biotechnol., 2017, 10, 250263.

19 F. Zaknoon, H. Sarig, S. Rotem, L. Livne, A. Ivankin, D. Gidalevitz and A. Mor, Antimicrob. Agents Chemother., 2009, 53, 3422-3429.

20 A. V. Presečki and D. Vasić-Rački, Biotechnol. Lett., 2005, 27, 1835-1839. 